\title{
HERITAGE-LED ONTOLOGIES: DIGITAL PLATFORM FOR SUPPORTING THE REGENERATION OF CULTURAL AND HISTORICAL SITES
}

\author{
BEATRICE TURILLAZZI ${ }^{1}$, GIOVANNI LEONI ${ }^{1}$, JACOPO GASPARI $^{1}$, ERNESTO IADANZA ${ }^{2}$, \\ MARINELLA MY ${ }^{3}$, MARTINA MASSARI ${ }^{1}$, SAVERIA O. M. BOULANGER ${ }^{1}$ \& AMIR DJALALI ${ }^{1}$ \\ ${ }^{1}$ Department of Architecture, University of Bologna, Italy \\ ${ }^{2}$ Inception Srl, Italy \\ ${ }^{3}$ Corvallis SpA, Italy
}

\begin{abstract}
The increasing application of digital technologies to cultural heritage $(\mathrm{CH})$ is wide and well documented, including a variety of tools such as digital archives, online guides and HBIM repositories. Several vocabularies and ontologies were designed to order heritage data and make $\mathrm{CH}$ more accessible and exploitable. However, these tools have often focused on a particular dimension of $\mathrm{CH}$ producing high value in separate sectors (e.g. access to conservation of historic buildings and data valorisation for restoration of heritage assets) but lacking ways for adapting or replicating the model to urban complex systems. Moreover, many studies and tools show large effort in cataloguing and archiving, but less in providing tools for designing and managing. The ROCK platform, developed within the Horizon 2020 (H2020) funded project ROCK (GA 730280), addresses the need for a management and interventionoriented interoperable tool, aimed at storing, visualizing, elaborating and linking data on cultural heritage. The use of already existing ontologies was not sufficient for developing a tool to deal with the complexity of urban systems and heterogeneous data sources. Instead, a participative methodology was set in place for the development of a context-based semantic framework to define the needs and requirements of heritage-led regeneration actions.

Keywords: semantic platforms, cultural heritage integrated management, sustainable development, heritage-led regeneration, living lab.
\end{abstract}

\section{INTRODUCTION}

In a context of great global changes, increasing urbanization and technological advancements, historical cities and cultural heritage $(\mathrm{CH})$, have been put forward since early 1990s as fundamental tools for sustainable and inclusive growth and development provided through urban regeneration processes [1]-[3]. CH-led urban regeneration processes assume heritage and its contextual characteristics as both repositories and engines of socio-economic development [4]-[6]. CH's "inherited legacies" [7] are assumed as unlimited source of knowledge and information to build a conscious design and planning for the present and future European built environment [8], a "heritage approach to growth" [9]. This information must be returned and made available in a clear manner to all actors who can put in place knowledge and skills to strengthen its value, and to those who can benefit from it. The necessity for making this data available for future uses demands new applications to facilitate information access through three-dimensional databases, without compromising the quality and amount of information captured in the survey [10] is one of the core concept of ROCK project, funded under the umbrella of Horizon 2020 programme (GA 730280).

Stemming from these premises, increasing accessibility to information in $\mathrm{CH}$-led regeneration processes leads to: 
- a recognition and classification of cultural heritage assets, in order to increase the understanding of its meanings and layers;

- promoting the involvement of various stakeholders, actors, formal or informal institutions carrying out independent agendas that need to be coordinated; and

- enabling informed decisions for the management and control of heritage maintenance and conservation interventions.

Addressing the issue of accessibility of $\mathrm{CH}$ provides a new perspective for considering it as a driver for the construction of more conscious urban regeneration processes, which intertwine development and economic growth with its conservation and enhancement.

Accessibility to $\mathrm{CH}$ and its inherent knowledge is increasingly extended by the possibilities provided by new technologies and tools developed over the years. Digital guides, augmented reality, online archives [11], [12] and digital platforms, management dashboards and monitoring sensors [13]-[16] are just few examples of the multitude of tools that are nowadays available for managing and designing interventions on $\mathrm{CH}$.

In this perspective, technologies and new digital media are increasingly adopted for promoting $\mathrm{CH}$ accessibility, collecting local material and immaterial data and gathering transnational knowledge, oriented to users, cultural operators and policy makers at once. A new set of technologies have made it possible not only to store historicised data, but also to dynamically monitor processes involving $\mathrm{CH}$ and to generate new data whose origin is diversified and fragmented. They can provide new opportunities for the "creation of new spaces, not only for consumption of heritage but also for its critical reflection" [17].

The Declaration "Cooperation on advancing digitization of cultural heritage" signed by Member States during the Digital Day 2019, has strengthened the commitment by the European Council, the European Parliament and the European Commission in promoting digital technologies to record, document and preserve Europe's $\mathrm{CH}$ and its accessibility to European citizens. Moreover, the Declaration states "The Union also needs to ensure that its digitised cultural content and related applications are available, where appropriate, on European platforms, in line with our values" [17, p. 2]. Furthermore, during the "Symposium Horizons for Heritage Research - Towards a Cluster on Cultural Heritage" of March 2019, the European Year of Cultural Heritage Team leader Dorothea Nigge highlighted the need to mobilise knowledge and research as a way to support advanced digitisation and foster actions for the future participation and access to $\mathrm{CH}$.

In this scenario, scholars, researchers, professionals, curators as well as city users residents, tourists, workers - have growing possibilities to interact with information and knowledge about different $\mathrm{CH}$ assets, to exchange experiences, to enrich data and contents through platforms and social media. This approach, together with the interpretation of heritage as an engine of urban regeneration, has produced a series of in-depth studies on common languages for the representation and accessibility of $\mathrm{CH}$, by developing a series of ontologies providing specialized and non-specialized information and visualization of data locally. However, these studies have often been focused on a particular dimension of $\mathrm{CH}$ (e.g. access to conservation of historic buildings, data valorisation for restoration of heritage assets), producing high value in separate sectors but lacking opportunity to adapt the model to urban complex systems. In fact, since a historical urban system is subject to complex dynamics, it seems necessary to provide a comprehensive method to trace and return the evolution of the heritage-driven regeneration system and propose solutions for future development. The objective of this article is to report a systemic and multidimensional methodology developed within Horizon 2020 ROCK project. In order to define the 
background framework, a preliminary review of existing studies and projects on $\mathrm{CH}$ ontologies is provided.

\section{HERITAGE ONTOLOGIES: A REVIEW}

A constellation of tools and technological techniques are emerging to address complexity in $\mathrm{CH}$ regeneration by organising the existing information and data into new ontologies for $\mathrm{CH}$ access, as requested by the Digital Cultural heritage NETwork [3]. In computer science, ontologies are ways to explicitly specify some concepts. An ontology is therefore a common denomination and formal definition of the properties and interrelations of characteristics that exist for a particular domain [18]. The ontology presents, in a machine-readable form, the semantic relations among things, to represent the reality in terms of computer data. The variables that define a certain system are recognised and organised establishing relations between them, building a shared language. The complexity of sources, methods, disciplines and large amounts of data emerges as a key issue to define a standard ontology. Many studies have addressed this challenge, specifically in the $\mathrm{CH}$ domain, in the attempt to "associate complementary information from various dedicated systems" [19], integrating schemes, organising people, assets and places.

These experiences have been placing high emphasis in defining an interoperable system, able to integrate, address and exchange [18], [19] information among technicians, professionals, policy makers, scholars, but also end users [21], investors and city-makers. In the European context, this led to the design and implementation of digital data interoperable platforms to:

- promote interaction, exchange and cooperation between European public administrations;

- deliver European public services across national borders and sectors; and

- create tools able to ensure interoperability between legal instruments, business processes, information exchanges and components.

The interoperability issue fits one of the challenges of EU Digital Single Market, about digitizing European $\mathrm{CH}$, making it accessible online and preserving it for future generations. Some examples of these attempts can be taken as a reference to highlight the lessons learned and the gaps still to be filled.

The INCEPTION (Inclusive Cultural Heritage in Europe through 3D Semantic Modelling) project offers an example of how to turn the architectural space as the foundation to create a protocol for optimizing the 3D documentation of $\mathrm{CH}$ [22]-[24]. Its platform addresses the spatial nature of $\mathrm{CH}$, standardising the survey, storage and retrieval of data of the physical consistency of heritage building and sites. This was made possible through the development of a semantic ontology dedicated to $\mathrm{CH}$ buildings, together with a data model for cataloguing information (also referring to the Art and Architecture thesaurus developed by the Getty Research Institute). These semantic attributes are integrated with hierarchicallyorganised and mutually-aggregated 3D digital geometric models [25], [26] aiming to adapt the fruition of each building component to different user needs. This methodology is specifically based on geometric physical data to avoid fragmented information acquired by different sources and to facilitate data access and use.

The approach proposed by Acierno et al. [27] is an ontology-based model highlighting a synergic approach for the management of complex information related to the conservation of $\mathrm{CH}$. In this model the authors pursue a twofold aim, to semantically capture and represent the conservation processes of $\mathrm{CH}$ and to put forward a model to exchange such information. What appears interesting is the domains in which the ontologies are modelled, which include 
the "actor" domain describing the people "concerned with the existence of the building" [27]. The model structures both direct and indirect knowledge and embeds also information emerging from stakeholders and users interested in the artefacts, even though it eventually focuses only on matters of conservation and restoration.

Kioussi et al. [28] work, aims to fill the existing gap on the lack of data on quality of materials and structures to be used as a background for decision-making in the selection of refurbishment strategies. This approach is oriented to develop criteria and methodologies to create a system for integrated documentation [28]. The scholars consider indicative parameters of data documentation, implementing national, European and international regulations to develop a common pan-European methodology governed by the existing European standards and codes [28]. The methodology proposes a quality control system based on three levels: (a) monitoring and inspection; (b) intervention; and (c) diagnosis.

Each level includes a set of data that cover aspects from functionality to interventions compatibility. The output of the methodology allows to move from the integrated documentation protocol to the parametrisation of the state of conservation [28] of each building based on the knowledge acquired on the asset during its lifetime. The application of the methodology provides the criteria and parameters that enable the selection in conservation problems and help to prioritize the monuments' needs, therefore producing an European $\mathrm{CH}$ identity card [29].

The aforementioned examples show that a great amount of data is nowadays produced by different sources, using IT technologies, social media, urban monitoring tools, environmental sensing, and the surveying activities carried on by public administrations and private corporations. In this framework, the issues of availability, accessibility, and transparency of information are the key elements for any inclusive, sustainable and informed $\mathrm{CH}$ fruition and $\mathrm{CH}-\mathrm{led}$ urban regeneration process.

\section{$3 \mathrm{CH}$, ONTOLOGIES AND COMPLEX URBAN SYSTEMS}

These studies are undoubtedly of considerable interest in addressing the issue of accessibility and interoperability of data and information on $\mathrm{CH}$. However, most of them are referred to individual buildings or to limited sites, often adopting a mono-dimensional methodological approach which refers to separate and sectoral indicators. This can't be fully transferred to large processes of urban regeneration in historical context whose layered and complex nature [9] require more comprehensive and cross-disciplinary approaches especially when intertwined with sustainable development [30].

Despite the growing efforts spent to boost the fruition and dissemination of $\mathrm{CH}$, what emerges is a lack of studies and tools addressing the organisation of information and data for the management and deployment of $\mathrm{CH}$-led regeneration, especially at urban scale. Many studies deal with cataloguing and archiving but less with providing tools for designing and managing. At the same time, they mostly focus on the "reconstruction of possible pasts" [19], overlooking the identification of hypothesis for future development. A series of gaps are highlighted both in the instruments, their characteristics and their performances concerning the accessibility of information on $\mathrm{CH}$ and its management in a wider urban perspective.

- Data and information on $\mathrm{CH}$ are growing in number, refining and diversifying, going to characterize heritage with intangible elements or linked to technical information on the genesis of the product or good itself. The great diversity of data available necessarily refers to different sources, fragmented databases, heterogeneous storage, access modes and languages that are often not comparable. 
- The ownership of the data is managed by different subjects or bodies, institutional or otherwise, a segmented constellation in which the actors involved are focused on their internal processes and struggle to interact with each other.

- The available tools and procedures are not able to cope with the complexity deriving from the not standardised information that $\mathrm{CH}$ has been accumulating and storing. They are unable to return a model that can recognise, describe, evaluate and replicate the vast amount of data surrounding $\mathrm{CH}$ both technical and qualitative, with an urban horizon.

What is evident is a lack of a shared vision for the collection, aggregation and process of data on complex dynamic systems such as the historic urban space, together with the narration and sharing of information on heritage monuments, buildings and entire sites at the urban scale. Historical urban space is in fact conceived as a whole, a collective system of spaces supporting all kind of relationships and connectivity at different levels. In order to enable urban regeneration processes and historical sites enhancements, $\mathrm{CH}$ should be conceived as a system of common objects, spaces and practices. Digital tools can help in the construction of such a model, allowing the mapping of formal and informal knowledge, practices, ideas and subjects, creating a collective regeneration resource for the city.

The ROCK (Regeneration and Optimisation of Cultural heritage in creative and Knowledge cities) H2020 project aims to tackle the aforementioned issues by granting the possibility to achieve interoperable models able to enrich the interdisciplinary knowledge of European cultural identity.

In the attempt to respond to these gaps, the ROCK project proposes an "enabling platform" [31], [32] for the knowledge, management, monitoring, redevelopment and enhancement of $\mathrm{CH}$.

\section{ROCK'S CH-LED REGENERATION APPROACH}

The ROCK project aims to support the transformation of historic city centres afflicted by physical decay, social conflicts and poor life quality into Creative and Sustainable Districts through shared generation of new sustainable environmental, social, economic processes. ROCK develops three integrated management plans for three test-bed sites in the cities of Bologna, Lisbon and Skopje, aggregating shared principles, local strategies with site-specific $\mathrm{CH}$-led regeneration tactics. ROCK provides new ways to access $\mathrm{CH}$ and to promote transparency and perception of collective property (as shared heritage), fostering the accessibility to spaces for all, improving the functions of $\mathrm{CH}$ from a user perspective, defining key policy issues, integrating the emerging spatial, temporal and virtual structures to support cohesion and develop a sense of belonging to a place [12].

ROCK promises to offer a possibility of thematic and systematic access to $\mathrm{CH}$-related data, its constraints and limitations as well as possibilities for new uses. Much of the information provided by ROCK derives and is shared by the intersectoral actors involved during the research activities. These elements are held together by a digital infrastructure, an interoperable platform based on the model of management dashboards typical of many smart city interfaces. With respect to the latter, ROCK intends to put together both the aggregated data and the elements that emerge from participatory processes related to the regeneration of $\mathrm{CH}$, in a decision support tool not only meant to be informative or managerial, but allowing the observation and the evaluation of a new approach to the regeneration of $\mathrm{CH}$ in European historical centres.

The ROCK platform operates on the relational aspects of $\mathrm{CH}$ recognition, production and management, providing procedures to design, monitor and evaluate $\mathrm{CH}$-led urban regeneration processes that includes and intertwines not only measurable data, but also 
information emerging from field observations, interviews, ethnographic research and participatory activities.

The platform defines a protocol, a common vocabulary, that allows a transition from a segmented approach - in which the actors involved are focused on their internal processes and the collected data are fragmented - to a circular one, where a digital ecosystem reconnects all actors involved in the $\mathrm{CH}$-driven regeneration process, enabling the exchange of information through the development of a conceptual grid made of historical data, empirical evidences and results from dynamic monitoring, to observe and validate the process.

The following section clarifies the methodological approach used by ROCK to identify the most useful datasets and interrelations to generate its ontology for $\mathrm{CH}$-led regeneration.

\subsection{Methodology}

The ROCK data platform is the tool through which a series of datasets are compared, visualised and interpreted through the development of a monitoring logic based on the needs emerged within Bologna's replicator site. What characterizes ROCK's approach is that the definition of the evaluation framework of the actions, and therefore the identification of the relevant datasets to be collected, was co-designed through the establishment of a dedicated panel, based on the model of the living lab. The local community was involved not only in order to collect information and knowledge about the site or to probe the needs and desires of the community for the regeneration of the area, but also in the definition of the main issues to address and look for in the definition and evaluation of the actions related to the regeneration process. The Bologna living lab - U-Lab, coordinated and facilitated by the local urban agency Fondazione Innovazione Urbana - saw the participation of representatives from several institutions, cultural producers, SMEs and citizen's association involved in the cultural life of the test-bed site, namely the University district [33].

U-Lab participants were firstly asked to react over the proposed definitions of "sustainability", "accessibility" and "collaboration for new cultural productions". In a second phase, the participants were asked to discuss these topics in the context of four selected locations in the university area. In this way, the laboratory produced three contextual definitions of the three topics (scenarios) and their various dimensions and articulations, also in relation to the actual locations, as well as the requirements to be met with the implementation of the regeneration actions.

The work of U-Lab produced a first shared vocabulary, a situated "ontology" (albeit not yet formalised into a digital tool) of urban regeneration for the Bologna University district, in relation to the principles of the ROCK project. This provides a contextual vocabulary where the definition of the scenarios may differ from the conventional understanding. For instance, sustainability was more perceived in terms of care of public spaces rather than within its environmental implications (waste, $\mathrm{CO}_{2}$ emission reduction, etc.). Similarly, accessibility was approached not simply in reducing or removing physical barriers, but as the necessity to create an inclusive environment which is able to welcome any kind of people or subjects. The issue of collaboration, finally, was framed in the sense of widening the offer of cultural events by the promotion of small cultural producers, as well as acting on the audience to widen and differentiate the public, also to attract different visitors to the University area.

These concepts were then translated into a series of requirements and indicators defined for each scenario, to which the various datasets were correlated, including the specific demonstration activities carried on in the test-bed site during the ROCK Project [34]. The datasets included in the ROCK platform can be grouped in the following main typologies: 
- datasets already available from public institutions or private companies, such as points of interest, museum and theatres attendance, data from free-floating bike sharing services, etc;

- datasets regarding ROCK's activities, collected through databases specifically developed for the ROCK project (such as the ROCK Atlas and ROCKME, with data compiled by ROCK partners);

- datasets coming from real-time sensors tested within the ROCK project (temperature, humidity, noise, pollution, wind, flows and video neuroanalytics); and

- datasets related to specific analyses and activities, including mapping events, sentiment analyses related to rock actions, surveys, ethnographic researches etc.

The ROCK interoperable platform allows data to be gathered, processed and compared facilitating communication and relationships between different information sources.

\subsection{The ROCK Platform}

The ROCK platform aims at providing data and insights on $\mathrm{CH}$ and urban regeneration for the use of policy makers, researchers, cultural producers and businesses, as well as providing insights and information for the general public and civil society (Fig. 1).

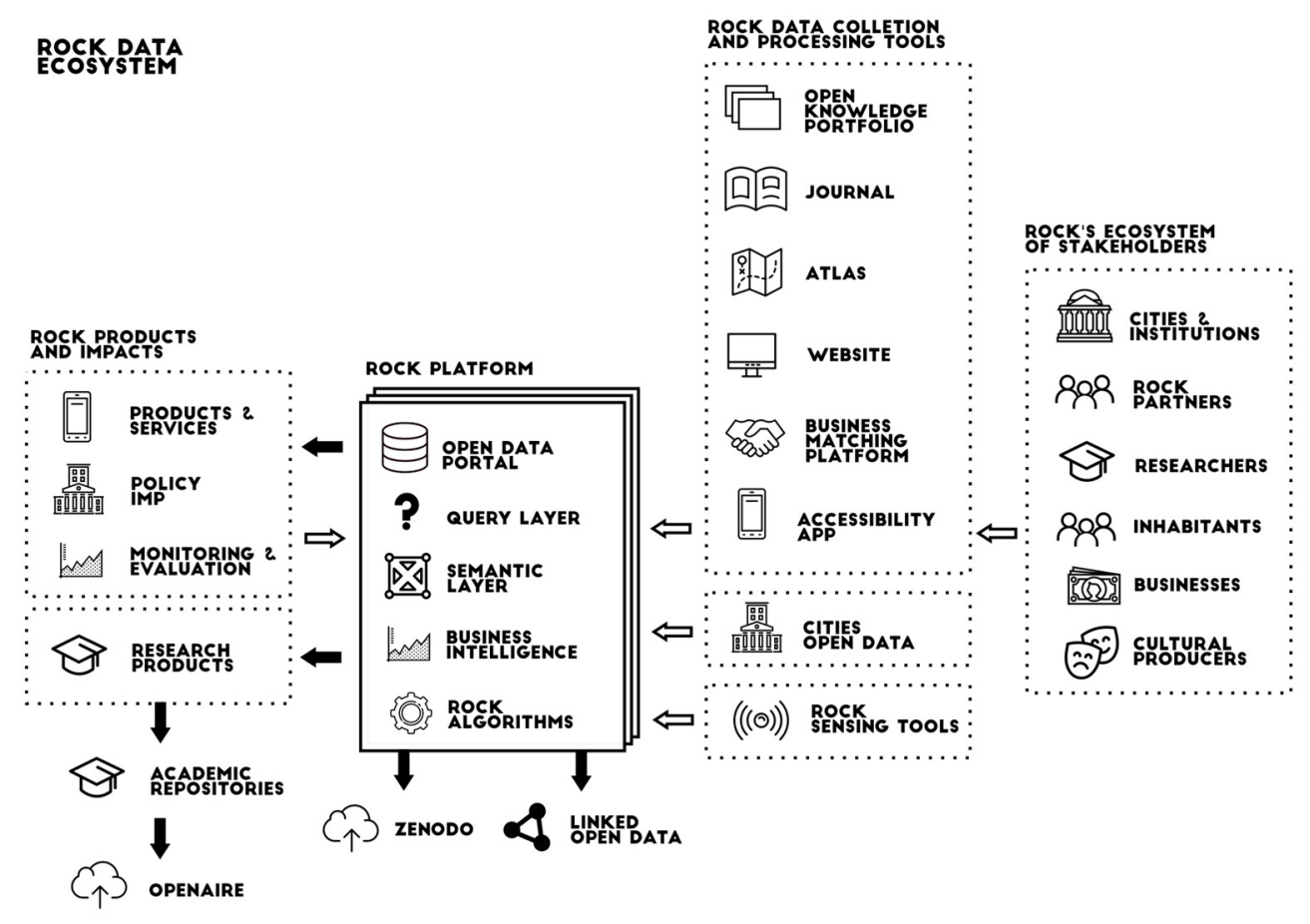

Figure 1: The ROCK tools' ecosystem.

The Platform is constituted by different layers specialized in performing specific functions (Fig. 2), interconnected with each other: the Query Layer, the Open Data Portal Layer, the Semantic Layer, the Business Intelligence Layer, the Big Data Layer. 


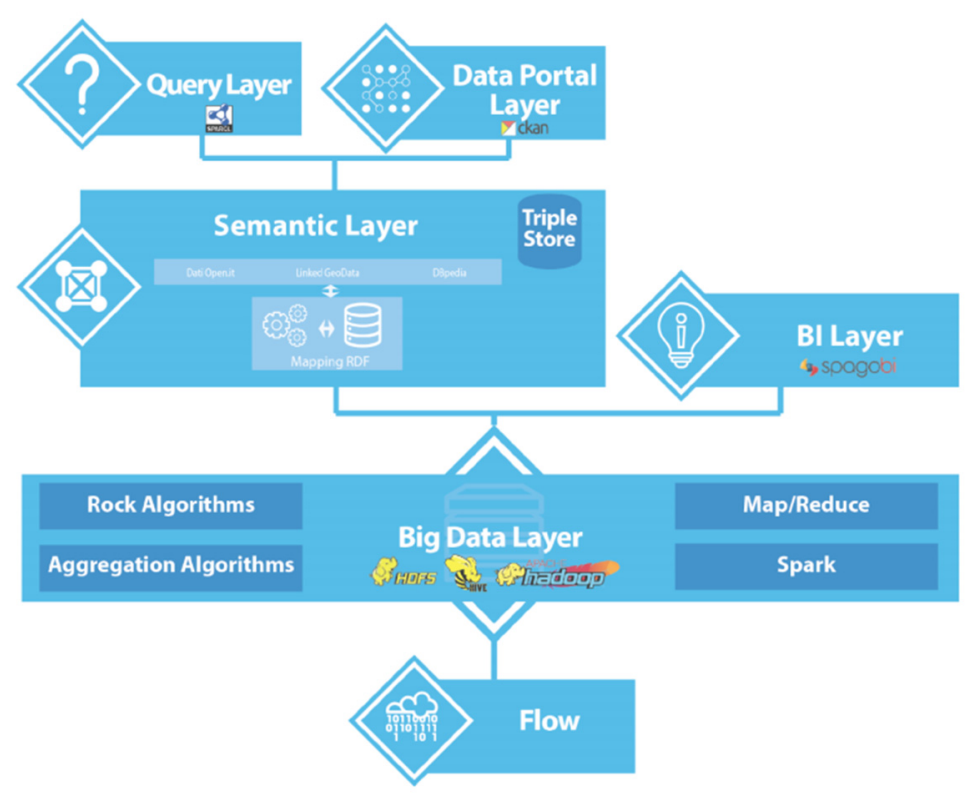

Figure 2: Platform layers.

The Open Data portal layer, based on CKAN, allows the storage and retrieval of data sets. Data are either uploaded directly by ROCK partners and stakeholders, or pulled through APIs (Application Programme Interfaces).

Data are then processed in the Semantic Layer, through a shared vocabulary and a knowledge based designed on existing standard ontologies for $\mathrm{CH}$ (e.g. the Getty Institute Art and Architecture Thesaurus, Link Geo Data Ontology, Dbpedia Ontology), tourism and points of interest, smart city sensors (e.g. SSN, Semantic Sensors Network Ontology). The use of semantic metadata allows not only to interoperate between heterogeneous data within the platform itself, but to link of related existing open data on $\mathrm{CH}$.

The Query Layer allows the query of the semantic database using a SPARQL endpoint. Both spatial and classical alphanumeric information can be expressed according to the model. The platform stores exemplary queries to allow the retrieval of data from users who are not familiar with SPARQL syntax. The Big Data layer contains the tools for processing the data provided, and it allows data aggregation, the calculation of KPIs, and the creation of different scenario to test and evaluate urban regeneration actions.

Finally, the Business Intelligence Layer consists in the definition of reports and dashboards (Fig. 3) for the interactive presentation and analytics on the available data from the different implemented tools of the project. The Business Intelligence Layer allows citizens, business players as well as public functionaries to have an immediate access to data, to gain insights on urban trends and create reports for presentation.

\section{CONCLUSIONS}

ROCK addresses the lack of a comprehensive approach to $\mathrm{CH}$ regeneration projects at urban scale, shifting the attention from individual objects, to the city as a multi-dimensional, spatiotemporal continuum produced by institutional and individual actions. The attention to 

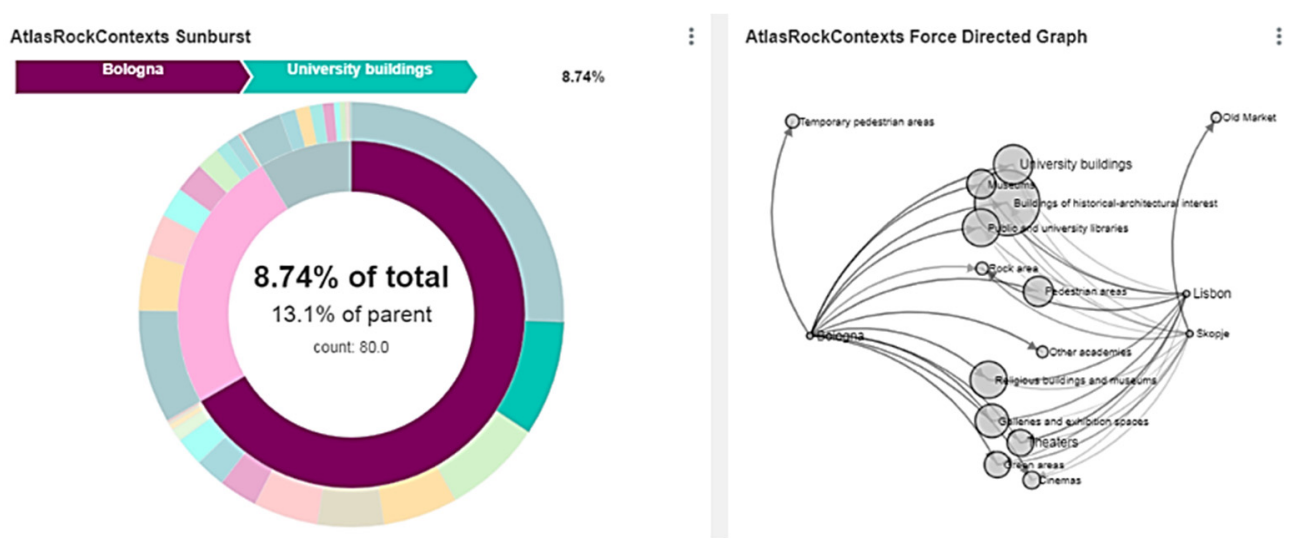

Figure 3: Example from the ROCK interoperable Platform's Business Intelligence Layer's possible visualisations.

heritage both at building and urban scale, allows the adoption of regeneration measures including new technologies, processes, new services and products to create new ways of recognizing, mapping appropriating $\mathrm{CH}$ while preventing socio-environmental decay and improving safety perception. The goal is to widen $\mathrm{CH}$ access and recognition, through a knowledge-based management to improve citizens' consciousness of $\mathrm{CH}$ as a common good that everybody can properly experience and must take care of. The scope is not simply to deliver intervention on the most representative $\mathrm{CH}$ elements, but to support and feed the creation of a sense of belonging where citizens become active actors of transformation, expressing their needs, expectations, visions to continue the evolution process within the city.

This is possible only providing a comprehensive and consistent picture of the available resources (material and immaterial ones), of the trends and possible synergies, of the institutional initiatives and network. This is the reason why the platform is so relevant in the project, providing a shared digital environment where all the involved subjects can contribute and at the same time can be informed of the progresses.

This approach requires the development of a common framework for the collection and reflection upon heterogeneous data, as well as the definition of qualitative and quantitative assessment methods to monitor and evaluate the impact of policies and actions in order to address architectural and urban design to inform urban policies. In the case of the ROCK project, this was made through an original use of the Living Lab paradigm, which involved citizens and stakeholders at different levels to co-design not only the actions to be undertaken, but also the tools to evaluate them in the framework of a common actions strategy. The results were necessarily linked to a specific environment and a cultural setting, influenced by local knowledge, languages, issues as well as the specific availability of resources. The use of the semantic approach allows the ROCK platform to be a scalable tool, capable of integrating information from various sources, including other $\mathrm{CH}$-related projects and new applications and services, being the platform accessible from external sources at the same time.

The proposed methodology is highly replicable in very different contexts and at different scales, as the other replicator cities are demonstrating within ROCK project, and it also provided an original conceptual understanding of sustainability, accessibility and collaboration, to engender further researches and design interventions in other contexts. 


\section{ACKNOWLEDGEMENT}

The ROCK research project (https://rockproject.eu/) is co-financed by the European Union within the H2020 framework programme with contract no. 730280 .

\section{REFERENCES}

[1] Townshend, T. \& Pendlebury, J., Public participation in conservation. Journal of Urban Design, 4, pp. 313-331, 1999. https://doi.org/10.1080/13574809908724453.

[2] UNESCO, The UNESCO recommendation on the historic urban landscape. http://unesdoc.unesco.org/images/0021/002150/215084e.pdf\#page=52. Accessed on: 30 Mar. 2019.

[3] DC-NET, Service priorities and best practices for digital cultural heritage. European Research Area Network, European Commission, Brussels, 2012.

www.digitalmeetsculture.net/article/dc-net-digital-cultural-heritage-network/.

Accessed on: 13 Mar. 2020.

[4] Reeve, A. \& Shipley, R., Heritage-based regeneration in an age of austerity: Lessons from the Townscape Heritage Initiative. Journal of Urban Regeneration and Renewal, 7(2), pp. 122-135, 2014. https://doi.org/10.1080/21650020.2014.893199.

[5] Boeri, A., Gaspari, J., Gianfrate, V., Longo, D. \& Pussetti, C., The adaptive reuse of historic city centres. Bologna and Lisbon: Solutions for urban regeneration. TECHNEJournal of Technology for Architecture and Environment, 12, pp. 230-237, 2016.

[6] Boeri, A. et al., Culture as primary political action in city governance: Three key concepts and ten policies to start with. European Journal of Creative Practices in Cities and Landscapes, 1, pp. 1-4, 2018.

[7] Meeks, S. \& Murphy, K.C., The Past and Future City: How Historic Preservation is Reviving America's Communities, Island Press: Washington, 2016.

https://doi.org/10.5822/978-1-61091-709-4.

[8] European Commission, Directorate-General for Research and Innovation, Getting cultural heritage to work for Europe. Report of the Horizon 2020 expert group on cultural heritage. https://ec.europa.eu/programmes/horizon2020/en/news/gettingcultural-heritage-work-europe. Accessed on 13 Nov. 2019.

[9] Fouseki, K. \& Nicolau, M., Urban heritage dynamics in "Heritage-led regeneration": Towards a sustainable lifestyles approach. The Historic Environment: Policy and Practice, 9(3-4), pp. 229-248, 2018. https://doi.org/10.1080/17567505.2018.1539554.

[10] Remondino, F., Heritage recording and 3D modeling with photogrammetry and 3D scanning. Remote Sensing, 3, pp. 1104-1138, 2011. https://doi.org/10.3390/rs3061104.

[11] Noh, Z., Sunar, M.S. \& Pan, Z., A review on augmented reality for virtual heritage system. International Conference on Technologies for E-Learning and Digital Entertainment, pp. 50-61, 2009. https://doi.org/10.1007/978-3-642-03364-3_7.

[12] Borowiecki, K.J., Forbes, N. \& Fresa, A., Cultural Heritage in a Changing World, Springer Science+Business Media, 2016. https://doi.org/10.1007/978-3-319-29544-2.

[13] Balzani, M. \& Maietti, F., The architectural space in an inclusive protocol for the 3D integrated acquisition of cultural heritage for documentation, diagnosis, representation, enhancement and conservation. The reasons of drawings. Thought, shape and model in the complexity management. Proceedings of the XXXVIII Convegno Internazionale dei Docenti della Rappresentazione - XIII Congresso Unione Italiana Disegno, eds S. Bertocci \& M. Bini, Gangemi Editore: Rome, pp. 1039-1044, 2016. 
[14] Bonsma, P. et al., Inception standard for heritage BIM models. Euro-Mediterranean Conference, Springer, Cham, IL, 2016.

[15] Iadanza, E. et al., Semantic web technologies meet BIM for accessing and understanding cultural heritage. 8th International Workshop 3D-ARCH $3 D$ Virtual Reconstruction and Visualization of Complex Architectures, Vol. 42, Copenicus, 2019.

[16] Bonsma, P. et al., Handling huge and complex 3D geometries with semantic web technology. IOP Conference Series: Materials Science and Engineering, 364(1), IOP Publishing, 2018.

[17] Cooperation on advancing digitisation of cultural heritage, Digital Day 2019, Brussels, Belgium, 9 April 2019. https://ec.europa.eu/digital-single-market/en/news/eumemberstates-sign-cooperate-digitising-cultural-heritage. Accessed on: 30 Apr. 2019.

[18] Doerr, M., Hunter, J. \& Lagoze, C., Towards a core ontology for information integration. Journal of Digital Information, 4(1), 2003.

[19] Doerr, M., Ontologies for Cultural Heritage. Handbook on Ontologies, Springer: Berlin, Heidelberg, pp. 463-486, 2009. https://doi.org/10.1007/978-3-540-92673-3_21.

[20] Stasinopoulou, T. et al., Ontology-based metadata integration in the cultural heritage domain. International Conference on Asian Digital Libraries, Springer: Berlin, Heidelberg, pp. 165-175, 2007. https://doi.org/10.1007/978-3-540-77094-7_25.

[21] Pattuelli, M.C., Modeling a domain ontology for cultural heritage resources: A usercentered approach. Journal of the American Society for Information Science and Technology, 62(2), pp. 314-342, 2011. https://doi.org/10.1002/asi.21453.

[22] Maietti, F., Di Giulio, R., Balzani, M., Piaia, E., Medici, M. \& Ferrari, F., Digital memory and integrated data capturing: Innovations for an inclusive cultural heritage in Europe through 3D semantic modelling. Mixed Reality and Gamification for Cultural Heritage, eds M. Ioannides, N. Magnenat-Thalmann \& G. Papagiannakis, Springer: Heidelberg, pp. 225-244, 2017.

https://doi.org/10.1007/978-3-319-49607-8_8.

[23] Maietti, F., Piaia, E. \& Turillazzi, B., Digital documentation: Sustainable strategies for cultural heritage assessment and inspection. SBE 16 MALTA International Conference - Europe and the Mediterranean - Towards a Sustainable Built Environment, Gutenberg Press: Malta, pp. 303-308, 2016.

[24] Maietti, F., Di Giulio, R., Piaia, E., Medici, M. \& Ferrari, F., Enhancing heritage fruition through 3D semantic modelling and digital tools: The INCEPTION project. IOP Conference Series: Materials Science and Engineering, 364(1), 012089, 2018. https://doi.org/10.1088/1757-899X/364/1/012089.

[25] Ioannides, M. et al. (eds), Digital heritage: Progress in cultural heritage Documentation, preservation, and protection. 6th International Conference, EuroMed, Springer: Cham, Switzerland, 2016. https://doi.org/10.1007/978-3-319-48496-9.

[26] Di Giulio, R., Maietti, F., Piaia, E., Medici, M., Ferrari, F. \& Turillazzi, B., Integrated data capturing requirements for 3D semantic modelling of cultural heritage: The INCEPTION Protocol. International Archives of the Photogrammetry, Remote Sensing and Spatial Information Sciences, 42(2/W3), pp. 251-257, 2017. https://doi.org/10.5194/isprs-archives-XLII-2-W3-251-2017.

[27] Acierno, M., Cursi, S., Simeone, D. \& Fiorani, D., Architectural heritage knowledge modelling: An ontology-based framework for conservation process. Journal of Cultural Heritage, 24, pp. 124-133, 2017. https://doi.org/10.1016/j.culher.2016.09.010. 
[28] Kioussi, A., Karoglou, M., Labropoulos, K., Bakolas, A. \& Moropoulou, A., Integrated documentation protocols able to support decision-making process in cultural heritage protection. Heritage protection from documentation to interventions, EU-CHIC International Conference on Cultural Heritage Preservation, eds R. Žarnic, V. Rajcic \& B. Vodopivec, Publ. University of Zagreb, Faculty of Civil Engineering: Ljubljana, pp. 129-131, 2012.

[29] Žarnić, R., EU-CHIC-European Cultural Heritage Identity Card, University of Ljubljana, Faculty for Civil and Geodetic Engineering, 2014.

[30] Boccardi, G. \& Duvelle, C., Background Note: Cultural Heritage and Sustainable Development: A Rationale for Engagement in UNESCO. Introducing Heritage into the Sustainable Development Agenda, ed. UNESCO, Hangzhou International Congress: Paris, 2013.

[31] Boeri, A., Gaspari, J., Gianfrate, V., Longo, D. \& Boulanger, S.O.M., Circular city: A methodological approach for sustainable districts and communities. Eco-Architecture VII: Harmonisation Between Architecture and Nature, 183, pp. 73-82, 2019. https://doi.org/10.2495/ARC180071

[32] Gianfrate, V., Djalali, A., Turillazzi, B., Boulanger, S.O.M. \& Massari, M., Researchaction-research towards a circular urban system for multi-level regeneration in historical cities: The case of Bologna. International Journal of Design and Nature and Ecodynamics, 15(1), pp. 5-11, 2020. https://doi.org/10.18280/ijdne.150102.

[33] Ginocchini, G. et al., U-Lab Incontri Tematici. http://dati.comune.bologna.it/download/file/fid/4465. Accessed on: 15 Nov. 2019.

[34] Topi, C. \& Cambridge, H., D4.1 Report on SMART KPIs and monitoring process. ROCK Project, 2019. 